\title{
Deep6mA: a deep learning framework for exploring similar patterns in DNA N6-methyladenine sites across different species
}

\author{
Zutan Li ${ }^{1 \#}$, Hangjin Jiang ${ }^{2 \#}$, Lingpeng Kong ${ }^{1}$, Yuanyuan Chen ${ }^{1}$, Liangyun Zhang $^{1^{*}}$, Cong Pian $^{1^{*}}$ \\ 1 Department of Mathematics, College of Science, Nanjing Agricultural University, Nanjing \\ 210095, China \\ 2 Center for Data Science, Zhejiang University, Hangzhou 310058, China \\ \# These authors are equally contributed to this work
}

* To whom correspondence should be addressed: piancong@njau.edu.cn and zlyun@njau.edu.cn.

\begin{abstract}
N6-methyladenin(6mA) is an important DNA modification form associated with a wide range of biological processes. Identifying accurately $6 \mathrm{~mA}$ sites on a genomic scale is crucial for understanding of $6 \mathrm{~mA}$ 's biological functions. In this paper, we developed, without requiring any prior knowledge of $6 \mathrm{~mA}$ and manually crafted sequence features, a deep learning framework named Deep6mA to identify DNA 6mA sites, and its performance is superior to other DNA 6mA prediction tools. Specifically, the 5-fold cross-validation on a benchmark dataset of rice gives the sensitivity and specificity of Deep6mA as $92.96 \%$ and $95.06 \%$, respectively, and the overall prediction accuracy is $94 \%$. Importantly, we find that the sequences with $6 \mathrm{~mA}$ sites share similar patterns across different species. The model trained with rice data predicts well the $6 \mathrm{~mA}$ sites of other three species: Arabidopsis thaliana, Fragaria vesca, and Rosa chinensis, with a prediction accuracy over $90 \%$. In addition, we find that (1) 6mA tends to occur at GAGG motifs, which means the sequence near the $6 \mathrm{~mA}$ site may be conservative; (2) $6 \mathrm{~mA}$ is enriched in the TATA box of the promoter, which may be the main source of its regulating downstream gene expression.
\end{abstract}

\section{INTRODUCTION}

DNA methylation modifications such as such as N4-methylcytosine $(4 \mathrm{mC})$, N6-methyladenine $(6 \mathrm{~mA})$, and 5-methylcytosine $(5 \mathrm{mC})$ play important roles in epigenetic regulation of gene expression without altering the sequence, and it is widely distributed in the genome of different species (1). DNA N6-methyladenine $(6 \mathrm{~mA})$ refers to the methylation of the 6th position of purine ring of adenine, which is one of the most abundant DNA modifications found in eukaryotes and prokaryotes (2). Researches on $6 \mathrm{~mA}$ shown that $6 \mathrm{~mA}$ plays important roles in DNA repair $(3,4)$, DNA replication (5), regulating gene transcription (6) and gene expression regulation (7). Although $6 \mathrm{~mA}$ sites are not uniformly distributed across the genome and they may be affected by environmental factors (8), the methylation protection is a genetic state, and $6 \mathrm{~mA}$ in prokaryotes and eukaryotes shows similar characteristics (9). It is indispensable to study the distribution of DNA $6 \mathrm{~mA}$ on the genome for a deeper understanding its epigenetic modification process.

Due to recent advances in high-throughput sequencing technologies, various experimental techniques were reported to promote the study of $6 \mathrm{~mA}$ distribution and its potential function in 
genome of eukaryotes and prokaryotes. For example, Pormraning et al. (2009) applied sequencing of methylated DNA immunoprecipitation technique to analyze genome-wide DNA methylation in eukaryotes. Krais et al. (2010) reported that the capillary electrophoresis with laser-induced fluorescence can be used to detect global adenine methylation in DNA. Meanwhile, Flusberg et al. (2010) used a method based on single-molecule, real-time sequencing technique to detect DNA methyladenine directly. Greer et al. (2015) used the ultrahigh-performance liquid chromatography and the mass spectrometry to discover DNA 6mA sites. These methods advanced the research of 6mA. By using 6mA-IP-Seq, Fu et al. (2015) found that $84 \%$ of $6 \mathrm{~mA}$ modification exit in Chlamydomonas genes. M. J. Koziol et al. (2016) identified the $6 \mathrm{~mA}$ modification in vertebrates by using HPLC, blots, and sequencing of methylated DNA Immunoprecipitation (MeDIP-seq). Zhou et al. (2018) found through $6 \mathrm{~mA}$ immunoprecipitation, mass spectrometry, and single molecule realtime that $0.2 \%$ of adenines in the rice genome are $6 \mathrm{~mA}$ methylated and GAGG-rich sequences are the most significantly enriched for $6 \mathrm{~mA}$.

To date, tools were developed to predict $6 \mathrm{~mA}$ methylation modification. For instance, Chen et al. (2019) proposed a method called i6mA-Pred to identify DNA 6mA sites based on the support vector (SVM) with 164 chemical features of nucleotides and position-specific nucleotide frequencies. The i6mA-Pred shows a good classification performance in rice $6 \mathrm{~mA}$ data. However, it does not fully capture the information between nearby nucleotides. To address this weakness, Pian et al. (2019) proposed to use MM-6mAPred, a first-order Markov model, to predict $6 \mathrm{~mA}$ sites. They found that there is a significant difference in the transition probabilities between the neighboring nucleotides in $6 \mathrm{~mA}$ sequences and non-6mA sequences. Compared with i6mA-Pred, MM-6mAPred has a better performance in terms of prediction accuracy.

The above two prediction models were trained on 880 rice $6 \mathrm{~mA}$ data and 880 non- $6 \mathrm{~mA}$ data and they did not consider the complex structure information in the sequence such as linkage disequilibrium between nucleotides, thus, there is still some room to improve. Zhou et al. (2018) found 265,290 of rice $6 \mathrm{~mA}$ data through a variety of experimental methods, such as HPLCMS/MS, 6mA immunoprecipitation sequencing and Single Molecule Real-Time (SMRT), which enables us to train complex models for $6 \mathrm{~mA}$ identification. For example, Lv et al. (2019) provided a new random forest model named iDNA6mA-rice based on the reconstructed $154,0006 \mathrm{~mA}$ data and 154,000 non-6ma data. iDNA6mA-rice is mainly realized by the random forest algorithm module (RF) based on three feature extraction techniques: K-tuple nucleotide frequency component, mono-nucleotide binary encoding and natural vector. Based on Convolutional Neural Networks (CNN), Yu and Dai (2019) proposed a method named SNNRice $6 \mathrm{~mA}$ to predict the $6 \mathrm{~mA}$ sites of rice, and showed its advantages over other methods. It is known to us that there is a strong dependence between nucleotides on the sequence, especially on the conserved DNA sequences, thus, the key difficulty in $6 \mathrm{~mA}$ prediction is to take into consider this dependence structure in statistical modeling. However, as we known, CNN is limited in learning information about long-distance dependence although it has a strong learning ability in the fields of image recognition, voice recognition and agricultural intelligence (10-13). Recurrent Neural Network (RNN) is a special neural network structure, inspired by the fact that human cognition is based on past experience and memory. Different from CNN, RNN not only considers the input of the previous moment, but also can effectively "remember" the previous content. Therefore, RNN has an advantage in analyzing the sequence containing timing 
information. At present, RNN has been widely used in fields such as natural language processing, image processing, machine translation, speech recognition and bioinformatics (1416). However, it is difficult to train an RNN due to gradient disappearance or gradient explosion. Long Short-Term Memory (LSTM) and Gate Recurrent Unit (GRU) are proposed to overcome this difficulty, and they are the most commonly used RNNs.

In this study, we introduced a novel deep learning framework named Deep6mA to identify DNA $6 \mathrm{~mA}$ sites. Deep6mA composed of a CNN and a bidirectional LSTM (BLSTM) module is shown to have a better performance than other methods on $6 \mathrm{~mA}$ prediction. Interestingly, we find that the motif with the highest frequency of $6 \mathrm{~mA}$ methylation is concentrated on GAGG among four species: Rice, Arabidopsis thaliana, Fragaria vesca, and Rosa chinensis, which means the $6 \mathrm{~mA}$ methylation has similar patterns across different species. This is further evidenced by the fact that the model trained by rice data has an accuracy over $90 \%$ to predict $6 \mathrm{~mA}$ in the other three species. We may conclude from these results that the sequence prone to DNA $6 \mathrm{~mA}$ methylation among different species is conservative, and Deep6mA may also be applicable to analyze the $6 \mathrm{~mA}$ site of other species. More importantly, we found that $6 \mathrm{~mA}$ is generally enriched in TATA box of the promoter. This may be an important way for $6 \mathrm{~mA}$ to regulate gene expression.

\section{MATERIAL AND METHODS}

\section{Benchmark dataset}

A benchmark dataset is important to build a reliable prediction model. In this study, for convenience, we use the $6 \mathrm{~mA}$-rice-Lv dataset $(17,18)$, including 154,000 positive samples and 154,000 negative samples, to evaluate the proposed method and to compare it with other methods. For each positive sample obtained from GEO, the sequence is $41 \mathrm{nt}$ long with the $6 \mathrm{~mA}$ site locating at the center. For each negative sample collected from NCBI, it's also a sequence with length $41 \mathrm{nt}$ but contains no $6 \mathrm{~mA}$ modification proved by experiments. In order to demonstrate that $6 \mathrm{~mA}$ shares the similar patterns across different species and our method can also be used to detect DNA 6mA sites of other species, we also collected DNA 6mA sequences of Arabidopsis thaliana, Fragaria vesca, and Rosa chinensis to show the ability of the trained Deep6mA from rice data on predicting 6mA methylation in these species. The 98483 $6 \mathrm{~mA}$ data of Arabidopsis thaliana is obtained from NCBI Gene Expression Omnibus (GEO) with accession number GSE81597, and the database MDR provides 26514 and 14677 6mA data for Fragaria vesca, and Rosa chinensis, respectively (http://mdr.xieslab.org/).

\section{Sequence representation}

Instead of using manually crafted DNA sequences features, we use the one-hot encoding method to convert the sequence into encoding tensor. Specifically, $\mathrm{A}, \mathrm{C}, \mathrm{G}, \mathrm{T}$, and $\mathrm{N}$ are encoded as $(1,0,0,0),(0,1,0,0),(0,0,1,0),(0,0,0,1)$, and $(0,0,0,0)$ respectively. Here the letter ' $\mathrm{N}$ ' represents a non-sequenced nucleotide. Thus, the input DNA sequence is represented as a 4 by 41 encoding matrix, and is viewed as an image which motivates our design of deep learning framework. 


\section{Convolutional neural network and long short term memory network}

Convolutional Neural Network (CNN) is widely used in image processing and speech recognition due to its high learning efficiency. The architecture of CNN is analogous to that of the connectivity pattern of neurons in the human brain and was inspired by the organization of the visual cortex. A CNN generally consists of three parts: convolution layers, pooling layers and fully connected layers, which enables it to successfully capture the spatial and temporal dependence in an image. The convolution layer extracts the high-level features such as edges, color and gradient orientation through multiple feature mapping. The resolution of feature mapping is compressed further by a pooling layer to extract dominant features which are rotational and positional invariant, and to decrease the computational power required to process the data. There are two types of Pooling: Max Pooling returning the maximum value from the portion of the image covered by the Kernel and Average Pooling returning the average of all the values from the portion of the image covered by the Kernel. Max Pooling tends to discard the noisy activations and performs de-noising along with dimension reduction; thus, it performs a lot better than Average Pooling. After multiple convolution and pooling processes, one or more fully connected layers are added to learn the non-linear combinations of high-level features from the convolutional layer.

Although $\mathrm{CNN}$ is powerful in image processing, however, it does not consider the dependence between inputs, and has a low power in sequence analysis such as natural language processing. Recurrent Neural Network (RNN) is proposed to overcome this shortcoming. As a special type of RNN, Long Short Term Memory Network (LSTM) is not only designed to capture the long dependent information in sequence but also overcome the training difficult of RNN due to gradient explosion and disappearance (19), thus it is the most widely used RNN in real applications. In LSTM, a storage mechanism is used to replace the hidden function in traditional RNN, with a purpose to enhance the learning ability of LSTM for long-distance dependency. Generally, an LSTM unit consists of a storage unit, a forgetting gate, an input gate and an output gate. Bi-directional LSTM (BLSTM), compared with unidirectional LSTM, captures better the information of sequence context.

\section{The Deep6mA Model}

The loci in DNA sequence are known to have a strong linkage disequilibrium, however, it is difficult to take into consider the dependence structure in traditional modeling for predicting $6 \mathrm{~mA}$ sites. In this section, to fully capture the information in the sequence, we introduce a deep learning network, Deep6mA, which has a CNN to extract high-level features in the sequence and a BLSTM to learn dependence structure along the sequence. Specifically, Deep6mA is consist of five layers of CNN, one BLSTM layer and one fully connected layer. The convolution layer in CNN has 256 filters with filter size 10. The activation function of CNN layers is the exponential linear unit (ReLU).

$$
\operatorname{ReLU}(\mathrm{x})= \begin{cases}0, & \text { if } \mathrm{x}<0 \\ \mathrm{x}, & \text { else }\end{cases}
$$

where $x$ is the feature map from the convolution operation. By viewing the input sequence as an image (see Section 2.2), the first convolution layer plays a role as motif detector of the $6 \mathrm{~mA}$ 
sites in genome, while the other convolution layers capture higher-level features underlying the sequence. After each convolution layer, a pooling layer with Max Pooling is added to reduce the redundancy of the features from the convolution layer. Then, one BLSTM layer with size 32 is added after CNN to learn the dependence structure in the sequence. The activation function used in this layer is the tanh activation function. A Fully Connected (FC) layer with 32 hidden units is used to learn the non-linear combinations of high-level features from the previous layers. Finally, a sigmoid activation function is used to combine the outputs from the FC layer to make the final decision. Figure 1 shows the flowchart of Deep6mA.

The loss function for training Deep6mA is set as the binary cross-entropy measuring the difference between the target and the predicted output.

$$
\mathrm{L}(\mathrm{w})=-\sum_{i=1}^{N} y_{i} \log \left(y_{i}^{\prime}\right)+\left(1-y_{i}\right) \log \left(1-y_{i}^{\prime}\right)+\alpha\|w\|_{2}
$$

Where $y_{i}$ is the true label, $y_{i}^{\prime}$ is the corresponding predicted value from Deep6mA, and $\alpha\|w\|_{2}$ is a regularization term to avoid overfitting.

Deep6mA is trained by using Adam (20). Batch normalization and dropout (21) are applied after each convolutional procedure to accelerate training and avoid overfitting. When training the model, the dropout rate is set as 0.5 , the learning rate is set as 0.01 , and the reduced factor is set as 0.5 . In addition, the maximum training epoch and batch size is set as 50 and 256, respectively. We take $1 / 8$ of training data, about $10 \%$ of the whole dataset, as validation data, and use an early stopping strategy with patience 5 , which means the training process will stop when prediction performance did not improve on the validation set. The whole framework is implemented in Pytorch (https://pytorch.org).

\section{Prediction accuracy assessment}

To the best of our knowledge, there are four leading $6 \mathrm{~mA}$ identification algorithms: i6mA-Pred, SNNRice6mA, iDNA6mA-rice and MM-6mAPred. iDNA6mA-rice is an improved version of i6mA-Pred, and SNNRice6mA is shown to better than iDNA6mA-rice (Yu and Dai, 2019), so we compare Deep6mA with SNNRice6mA and MM-6mAPred. Four metrics, the prediction accuracy (ACC), sensitivity (SN), specificity (SP), and Matthews correlation coefficient (MCC), are used to evaluate the predictive performance of the methods discussed here. Their definitions are given below. A better method should have a higher value on ACC, SN, SP and MCC. The receiver operating characteristic curve (ROC), the area under the curve (11) and precision recall curves (PRC) are used to show the detailed performance of the methods discussed here. The $X$-axis of the ROC curve is false positive rate (FPR=1-SP), and the $Y$-axis is true positive rate (TPR=SN). The $X$-axis of the PRC curve is recall (Recall=SN), and the $Y$ axis is precision.

$$
\begin{gathered}
S_{N}=\frac{T_{P}}{T_{P}+F_{N}} \\
S_{P}=\frac{T_{N}}{T_{N}+F_{P}} \\
\text { Precision }=\frac{T_{P}}{T_{P}+F_{P}}
\end{gathered}
$$




$$
\begin{gathered}
A C C=\frac{T_{P}+T_{N}}{T_{P}+T_{N}+F_{P}+F_{N}} \\
M C C=\frac{T_{P} \times T_{N}-F_{P} \times F_{N}}{\sqrt{\left(T_{P}+F_{P}\right) \times\left(T_{N}+F_{N}\right) \times\left(T_{P}+F_{N}\right) \times\left(T_{N}+F_{P}\right)}}
\end{gathered}
$$

where TP is the number of real $6 \mathrm{~mA}$ sequences predicted correctly as $6 \mathrm{~mA}$ methylated, TN is the number of non- $6 \mathrm{~mA}$ sequences correctly predicted as non-6mA methylated, $\mathrm{FN}$ is the number of $6 \mathrm{~mA}$ sequences predicted incorrectly as non- $6 \mathrm{~mA}$ methylated and $\mathrm{FP}$ is the number of non- $6 \mathrm{~mA}$ sequences predicted incorrectly as $6 \mathrm{~mA}$ methylated.

\section{RESULTS}

\section{Comparing CNN with CNN+LSTM}

In this section, we compare the performance of CNN with CNN + LSTM based on the same training data under different settings of CNN. Note that we use the same structure of CNN to compare these two methods. The number and filter of convolution layer in CNN is set to 1 and 5 respectively, the corresponding convolution kernel size is set as 32, 64, 128 and 256, respectively, and unit size of LSTM is set to 32 . Table 1 is a comparison of the performance of these two methods under different convolution kernels. The result shows that the performance of CNN + LSTM is better than that of CNN, due to the ability of LSTM to learn the dependence structure underlying the sequence.

To better understand the performance of CNN and CNN+LSTM, we show in Figure 2 their ROC curve and PRC curve based on a CNN with 1 convolution layer, 64 convolution kernels, and filter size 10, and a LSTM with 32 hidden units. The AUC of CNN+BLSTM is 0.974 , which is 0.124 higher than that of the CNN. Meanwhile, the PR curve also shows that CNN + LSTM is better than CNN.

\section{Selecting model parameters of CNN+LSTM}

It is known that the performance of CNN+LSTM framework depends on the filter size and the number, convolution kernels of the convolution layer, and number of hidden units in LSTM. To simplify notations, we denote the CNN+LSTM framework with $x$ convolution layer(s), $y$ convolution kernel(s), $z$ filter size and $w$ hidden units as a CNN+LSTM with parameter $x-y-z-w$. In this section, we select the best CNN+LSTM model from 30 different settings of parameter $x$, $y, z$, w by 5 -fold cross-validation. Specifically, we take $x$ from $\{1,2,3,4\}$, y from $\{64,256,512\}$, $w$ from $\{16,32\}$ and fix $z$ at 10 . Figure 3 shows the prediction performance of CNN+LSTM model under different settings of parameters. According to these results, the CNN+LSTM with parameter 5-256-10-32 is chosen as our final CNN+LSTEM model.

\section{Comparison of motifs across different species}

Bailey and Elkan (1995) proposed a MEME algorithm to identify one or more motifs in the sequence based on a binary hybrid model and Expectation-Maximization (EM) algorithm. Here, 
we use MEME to compare motifs from four species: Rice, Arabidopsis thaliana, Fragaria vesca and Rosa chinensis. From Figure 4, we see that GAGG is the most significantly associated motif in these four species. Hence, we infer that DNA 6mA methylation occurs most frequently at GAGG motifs across different species. Xiao et al. (2018) also pointed out that (G/C) AGG $(\mathrm{C} / \mathrm{T})$ is the most frequent motif in human genome. All these together suggest that the sequence near the DNA 6mA methylation site may be conserved among different species.

\section{Location features of $6 \mathrm{~mA}$ sites}

It is well known that 5 -methylcytosine $(5 \mathrm{mC})$ is one of the most important DNA methylation modifications. The results show that the determination of differential methylation region (DMR) is more biologically explanatory and statistically significant than that of the differential methylation sites measured separately (Chen, et al, 2017). Based on this, we expect to know which regions of the genome will be enriched by $6 \mathrm{~mA}$ methylation. Figure $5 \mathrm{~A}$ shows the distribution of distance between adjacent $6 \mathrm{~mA}$ site in 12 chromosomes. It can be seen from Figure $5 \mathrm{~A}$ that the distribution of $6 \mathrm{~mA}$ methylation modification on 12 chromosomes is consistent. The mean of the distance between adjacent $6 \mathrm{~mA}$ sites is greater than $26=64$, which means rare $6 \mathrm{~mA}$ sites occur in a continuous region like $5 \mathrm{mC}$ sites to form a DMR. To explore further the location features of $6 \mathrm{~mA}$ sites, we look inside the subsequences of length $30 \mathrm{nt}$ with more than five $6 \mathrm{~mA}$ sites, and find that almost all of the $6 \mathrm{~mA}$ sites in these subsequences are located in the TATA box of the promoter (see Figure 5B for example, more details are given in Supplementary Material). This implies that $6 \mathrm{~mA}$ is generally enriched in TATA box which is a very important functional component of the promoter. The transcription process will not start until RNA polymerase binds tightly on TATA box. Therefore, the enrichment of $6 \mathrm{~mA}$ methylation on TATA box may directly affect the transcription and expression of downstream genes. This may be an important regulatory function of $6 \mathrm{~mA}$ methylation modification.

\section{Comparison with other leading methods}

We use 5-fold cross-validation to evaluate the performance of Deep6mA, SNNRice6mA and MM-6mAPred based on 6mA-rice-Lv dataset (see Section 2.1). The settings of the iDNA6mArice and SNNRice6mA follow that given in the corresponding original paper. Specifically, the number of trees in forest algorithm (RF) in iDNA6mA-rice is set to 100 with the seed of 1 . For SNNRice6mA, the number of convolution layer and convolution kernel is set to 1 and 4 , respectively, and the filter size and fully connect layer size of SNNRice6mA is set to 3 and 64 , respectively. Deep6mA is the best one among these methods in terms of SN, SP, ACC and MCC as shown in Table 2 with SN, SP, ACC and MCC as 95.06\%, 92.96\%, 94.01\% and 0.88 respectively. The better performance of Deep $6 \mathrm{~mA}$ is mainly due to the ability of BLSTM to learn the dependence structure between distant nucleotides.

In addition, the ROC curve and PR curve of Deep6mA, SNNRice6mA and MM-6mAPred are shown in Figure 6. The area under curve of Deep6mA is 0.979 , which is higher than that from other two methods. All these results show that our method Deep6mA is the best one among these methods.

\section{Validation on other three species}


Results in Section 3.3 show that $6 \mathrm{~mA}$ is conservative among different species, which suggest that Deep6mA trained on rice data is applicable to predict the $6 \mathrm{~mA}$ sites of other species. In the following, we try to validate this principle by applying the trained Deep6mA to the $6 \mathrm{~mA}$ data of other three species: Arabidopsis thaliana with sample size 98483, Fragaria vesca with sample size 26514 and Rosa chinensis with sample size 14677 (see Section 2.1 for details). The prediction results on these three test datasets are listed in Table 3. We found that Deep6mA trained with rice $6 \mathrm{~mA}$ data predicts with high accuracy the $6 \mathrm{~mA}$ sites in other three species. The prediction performance of our method is better than SNNRice6mA and MM$6 \mathrm{mAPred}$. This makes deep6mA have great potential in predicting $6 \mathrm{~mA}$ sites in other species' genomes.

\section{DISCUSSION}

In this study, we propose a deep learning framework named Deep6mA by integrating CNN and LSTM to efficiently predict DNA 6mA sites. Deep6mA uses a CNN layer to extract DNA sequence characterization, and then spreads it into a BLSTM layer to capture context dependency information of $6 \mathrm{~mA}$ sites. Finally, these features are transferred to a fully connected layer to determine whether the site is a $6 \mathrm{~mA}$ site. The experimental results show that Deep6mA can predict the $6 \mathrm{~mA}$ site of rice with high accuracy. Importantly, we found that most of the $6 \mathrm{~mA}$ methylation modifications in different species are more likely to occur on GAGG motifs. This shows that DNA sub-sequences containing $6 \mathrm{~mA}$ sites among species have certain conservation. Maybe this is the reason why Deep6mA model trained with rice data can accurately predict $6 \mathrm{~mA}$ sites in other three species. However, there are some inadequacies in this study, such as the selection of sequence length. In theory, the longer the sequence, the more information it provides. All previous studies on $6 \mathrm{~mA}$ site recognition are based on the sequence with a length of $41 \mathrm{nt}$. It is not necessary to learn the complete sequence information by only training the model with those short sequences. Besides, due to the relative complexity of the calculation time, the framework and parameter design of deep6mA may only achieve a local optimum. What's more, why is the $6 \mathrm{~mA}$ site enriched in the TATA box of the promoter, and whether this enrichment has a regulatory effect on the expression of downstream genes. For the ongoing work, we will carry out further research on these issues

\section{DATA AVAILABILITY}

Source code, all training data and testing data of Deep6mA are found at https://github.com/Marscolono/Deep6mA

\section{SUPPLEMENTARY DATA}

Supplementary Data are available at NAR online.

\section{ACKNOWLEDGEMENT}

We would like to thank Prof. Xiaodan Fan from The Chinese University of Hong Kong for valuable discussions. 


\section{FUNDING}

This work is partially supported by Startup Foundation for Advanced Talents at Nanjing Agricultural University (No. 050/804009), The National Natural Science Foundation of China (No. 11901517), and The Fundamental Research Funds for the Central Universities (No. $1 \mathrm{~A} 3000 * 172210192)$.

\section{REFERENCES}

1. Ratel, D., Ravanat, J.L., Berger, F. and Wion, D. (2006) N6-methyladenine: the other methylated base of DNA. Bioessays, 28, 309-315.

2. Vanyushin, B.F., Tkacheva, S.G. and Belozersky, A.N. (1970) Rare bases in animal DNA. Nature, 225, 948-949.

3. Pukkila, P.J., Peterson, J., Herman, G., Modrich, P. and Meselson, M. (1983) Effects of high levels of DNA adenine methylation on methyl-directed mismatch repair in Escherichia coli. Genetics, 104, 571-582.

4. Au, K.G., Welsh, K. and Modrich, P. (1992) Initiation of Methyl-Directed Mismatch Repair. Journal of Biological Chemistry, 267, 12142-12148.

5. Campbell, J.L. and Kleckner, N. (1990) E. coli oriC and the dnaA gene promoter are sequestered from dam methyltransferase following the passage of the chromosomal replication fork. Cell, 62, 967-979.

6. Cheng, L., Sun, J., Xu, W.Y., Dong, L.X., Hu, Y. and Zhou, M. (2016) OAHG: an integrated resource for annotating human genes with multi-level ontologies. Scientific Reports, 6, 1-9.

7. Low, D.A., Weyand, N.J. and Mahan, M.J. (2001) Roles of DNA adenine methylation in regulating bacterial gene expression and virulence. Infect Immun, 69, 7197-7204.

8. Wion, D. and Casadesus, J. (2006) N-6-methyl-adenine: an epigenetic signal for DNA-protein interactions. Nature Reviews Microbiology, 4, 183-192.

9. Heyn, H. and Esteller, M. (2015) An Adenine Code for DNA: A Second Life for N6Methyladenine. Cell, 161, 710-713.

10. Abdel-Hamid, O., Mohamed, A.R., Jiang, H. and Penn, G. (2012) Applying Convolutional Neural Networks Concepts to Hybrid Nn-Hmm Model for Speech Recognition. Int Conf Acoust Spee, 4277-4280.

11. Alipanahi, B., Delong, A., Weirauch, M.T. and Frey, B.J. (2015) Predicting the sequence specificities of DNA- and RNA-binding proteins by deep learning. Nat Biotechnol, 33, 831-+.

12. Krizhevsky, A., Sutskever, I. and Hinton, G.E. (2017) ImageNet Classification with Deep Convolutional Neural Networks. Commun Acm, 60, 84-90.

13. Zhou, J. and Troyanskaya, O.G. (2015) Predicting effects of noncoding variants with deep learning-based sequence model. Nat Methods, 12, 931-934.

14. Ning, K., Ng, H.K., Srihari, S., Leong, H.W. and Nesvizhskii, A.I. (2010) Examination of the relationship between essential genes in PPI network and hub proteins in reverse nearest neighbor topology. Bmc Bioinformatics, 11.

15. Liu, S.J., Yang, N., Li, M. and Zhou, M. (2014) A Recursive Recurrent Neural Network for Statistical Machine Translation. Proceedings of the 52nd Annual Meeting of the Association for Computational Linguistics, Vol 1, 1491-1500.

16. Donahue, J., Hendricks, L.A., Rohrbach, M., Venugopalan, S., Guadarrama, S., Saenko, K. and 
Darrell, T. (2017) Long-Term Recurrent Convolutional Networks for Visual Recognition and Description. IEEE Trans Pattern Anal Mach Intell, 39, 677-691.

17. Zhou, C., Wang, C.S., Liu, H.B., Zhou, Q.W., Liu, Q., Guo, Y., Peng, T., Song, J.M., Zhang, J.W., Chen, L.L. et al. (2018) Identification and analysis of adenine N-6-methylation sites in the rice genome. Nature Plants, 4, 554-563.

18. Lv, H., Dao, F.Y., Guan, Z.X., Zhang, D., Tan, J.X., Zhang, Y., Chen, W. and Lin, H. (2019) iDNA6mA-Rice: A Computational Tool for Detecting N6-Methyladenine Sites in Rice. Frontiers in Genetics, 10.

19. Greff, K., Srivastava, R.K., Koutnik, J., Steunebrink, B.R. and Schmidhuber, J. (2017) LSTM: A Search Space Odyssey. Ieee Transactions on Neural Networks and Learning Systems, 28, 2222-2232.

20. Kingma, D. and Ba, J. (2014) Adam: A Method for Stochastic Optimization. Computer Science.

21. Srivastava, N., Hinton, G., Krizhevsky, A., Sutskever, I. and Salakhutdinov, R. (2014) Dropout: A Simple Way to Prevent Neural Networks from Overfitting. Journal of Machine Learning Research, 15, 1929-1958. 


\section{TABLE AND FIGURES LEGENDS}

Table 1. The performance of CNN and CNN + LSTM under different Convolution kernels

\begin{tabular}{|c|c|c|c|c|c|c|c|}
\hline & $\begin{array}{c}\text { Convolution } \\
\text { kernels }\end{array}$ & LSTM size & SN (\%) & SP (\%) & $\operatorname{ACC}(\%)$ & MCC & AUC \\
\hline \multirow{4}{*}{$\sum_{z}^{\Omega}$} & 32 & 0 & 76.01 & 78.01 & 77.01 & 0.54 & 0.85 \\
\hline & 64 & 0 & 74.24 & 79.95 & 77.1 & 0.55 & 0.85 \\
\hline & 128 & 0 & 68.77 & 83.41 & 76.1 & 0.53 & 0.85 \\
\hline & 256 & 0 & 69.34 & 83.73 & 76.53 & 0.54 & 0.86 \\
\hline \multirow{4}{*}{ 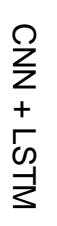 } & 32 & 32 & 95.32 & 90.08 & 92.7 & 0.86 & 0.97 \\
\hline & 64 & 32 & 95.5 & 90.61 & 93.05 & 0.86 & 0.97 \\
\hline & 128 & 32 & 95.62 & 91.28 & 93.45 & 0.87 & 0.97 \\
\hline & 256 & 32 & 95.24 & 91.73 & 93.48 & 0.87 & 0.97 \\
\hline
\end{tabular}

Table 2. Comparison of Deep6mA, SNNRice6mA and MM-6mAPred based on 6Ma-rice-Lv dataset.

\begin{tabular}{llllll}
\hline Method & SN (\%) & SN (\%) & ACC (\%) & MCC & AUC \\
\hline Deep6mA & 95.06 & 92.96 & 94.01 & 0.88 & 0.98 \\
SNNRice6mA-large & 94.33 & 89.75 & 92.04 & 0.84 & 0.97 \\
MM-6mAPred & 93.47 & 89.51 & 91.49 & 0.83 & 0.96 \\
\hline
\end{tabular}

Table 3. Prediction accuracy of Deep6mA, SNNRice6mA and MM-6mAPred trained with rice data on Arabidopsis thaliana, Fragaria vesca and Rosa chinensis.

\begin{tabular}{cccc}
\hline Method & Fragaria vesca (\%) & Rosa chinensis (\%) & Arabidopsis thaliana (\%) \\
\hline Deep6mA & 93.40 & 90.99 & 98.17 \\
SNNRice6mA & 90.94 & 89.03 & 91.18 \\
MM-6mAPred & 89.43 & 90.69 & 93.48 \\
\hline
\end{tabular}


Figure 1. The flowchart of Deep6mA. The structure of Deep6mA consists of five layers of CNN, one layer of BLSTM and one layer of full connection layer.

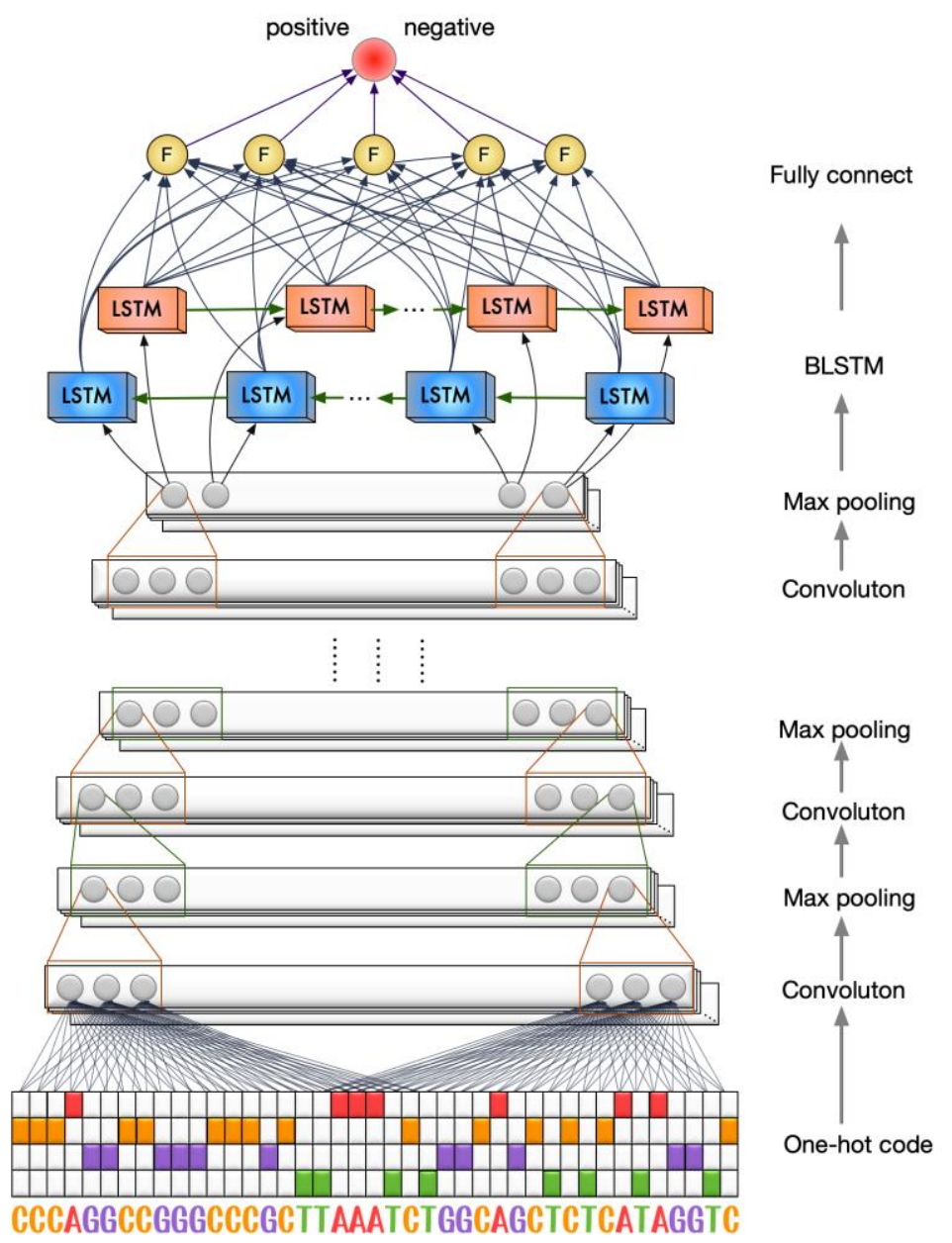

Figure 2. The ROC and PRC curve of CNN and CNN + LSTM based on CNN with 1 convolution layer, 64 convolution kernels, 32 hidden units of LSTM and filter size 10.

A

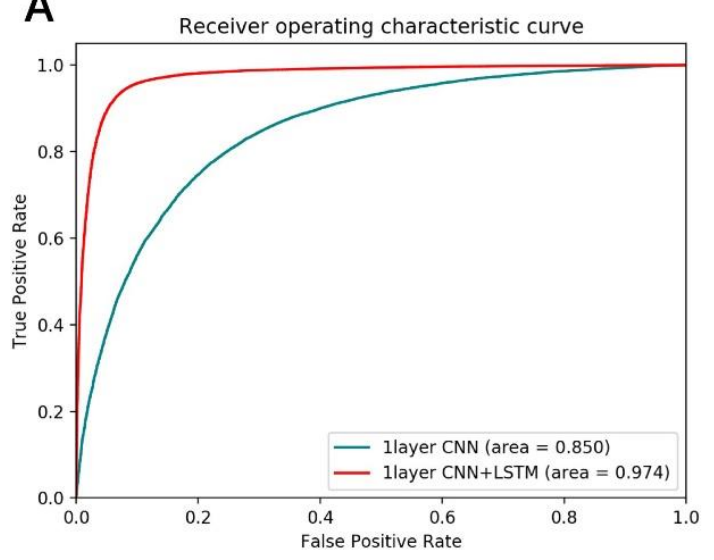

B

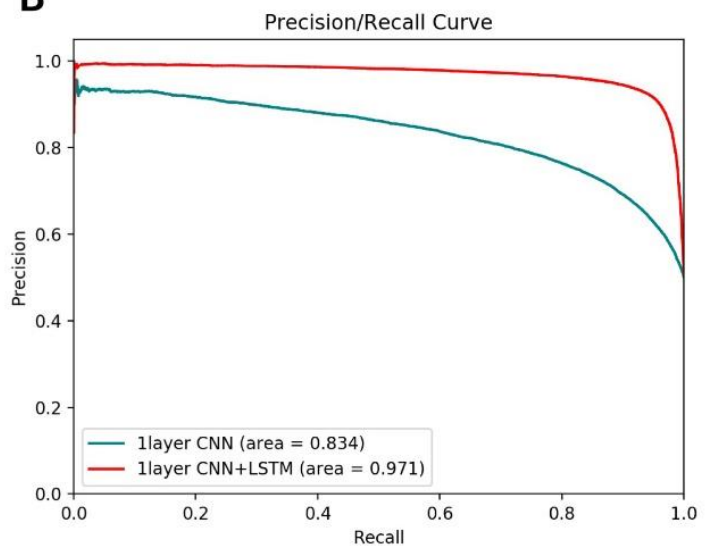


Figure 3. Prediction performance of CNN+LSTM model under different settings of parameters. The CNN+LSTM with parameter 5-256-10-32 is chosen as our final CNN+LSTM model (convolution layer: 5 , convolution kernel: 256 , filter size: 10 , hidden units of LSTM: 32 ).
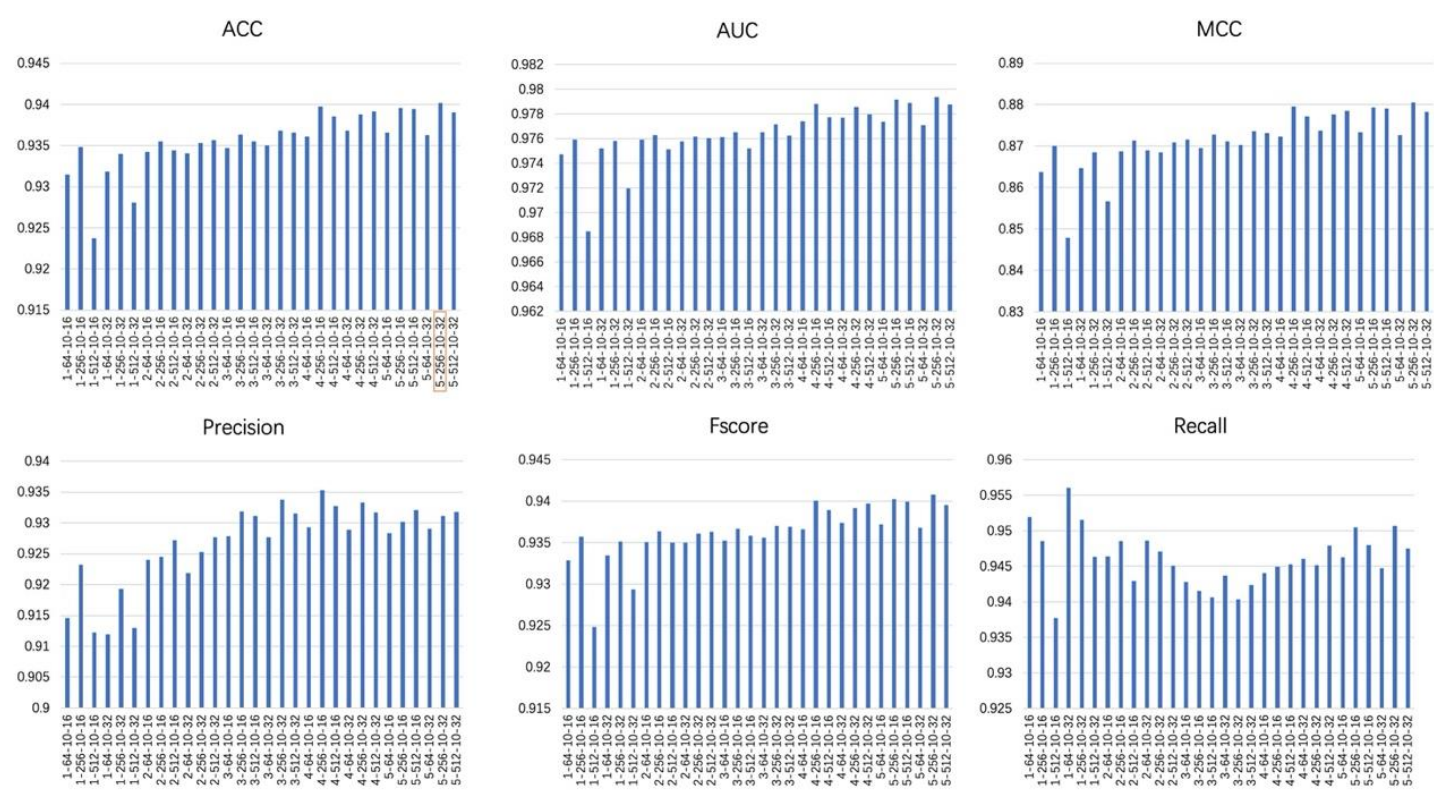

Figure 4. The top two significant motifs in the sequence near the $6 \mathrm{~mA}$ site of four species (A: Arabidopsis thaliana, B: Rosa chinensis, C: Rice, and D: Fragaria vesca).

A

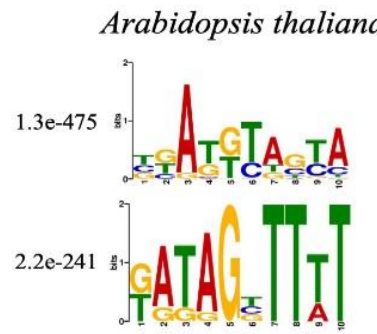

$\mathrm{C}$

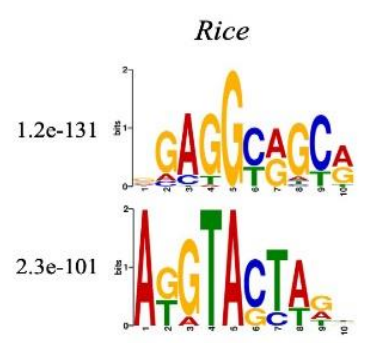

B Rosa chinensis

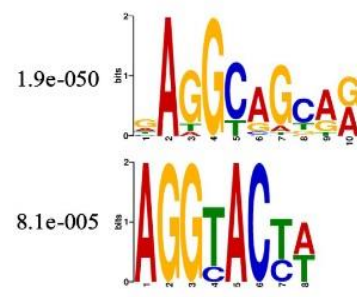

$\mathrm{D}$

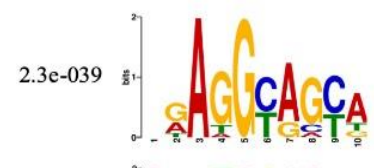
1.1e-003 
Figure 5. Location features of $6 \mathrm{~mA}$ sites in the sequence. (A) The distribution of $6 \mathrm{~mA}$ methylation modification on 12 chromosomes. The $\mathrm{X}$ axis and $\mathrm{Y}$ axis represent the 12 chromosomes and the logarithm of distance between adjacent $6 \mathrm{~mA}$ site. (B) Three examples of sequences containing TATA box enriched with $6 \mathrm{~mA}$ methylation (colored in red).

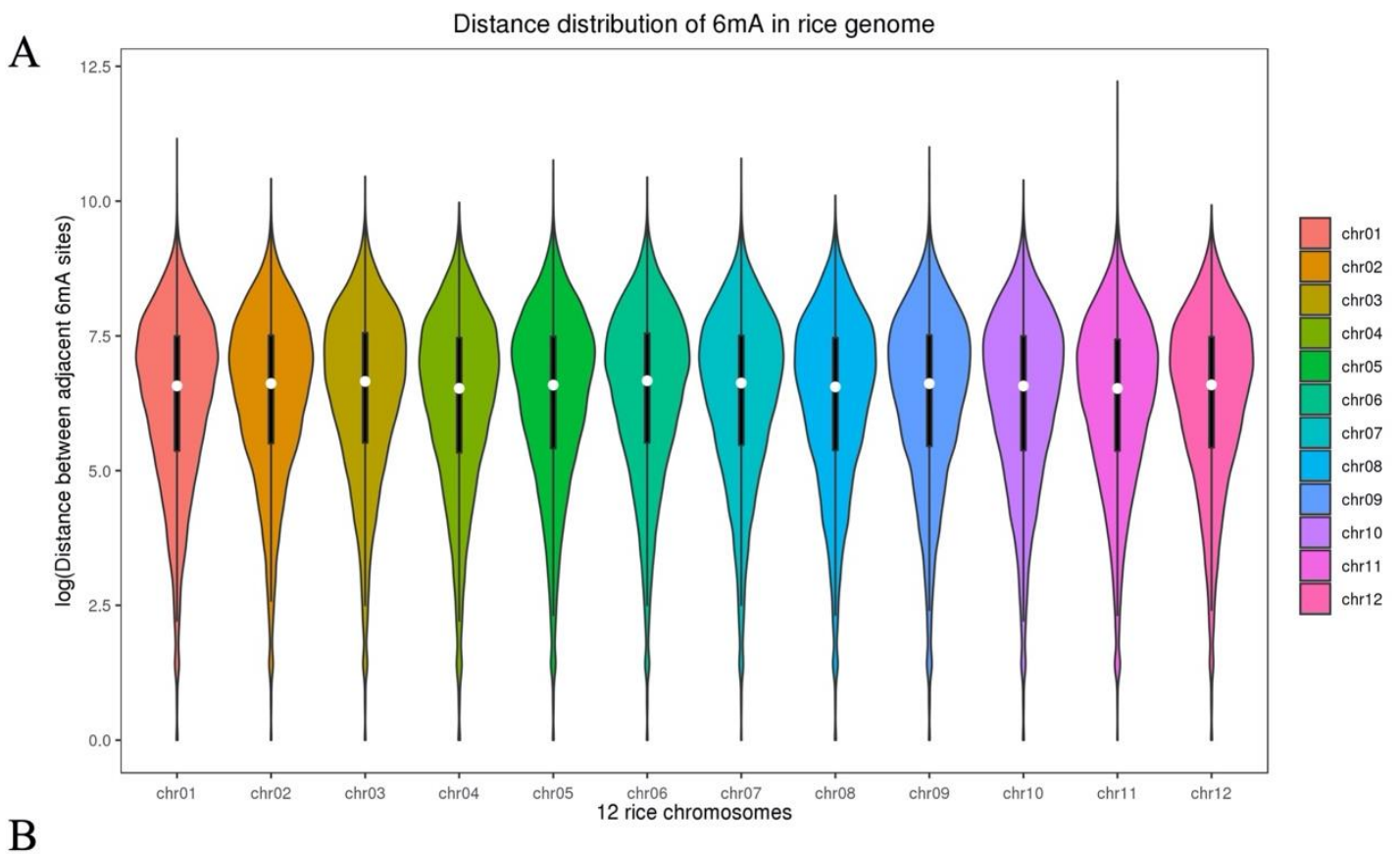

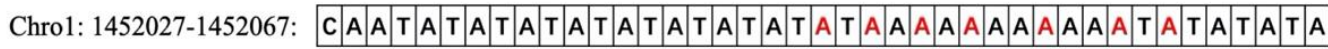

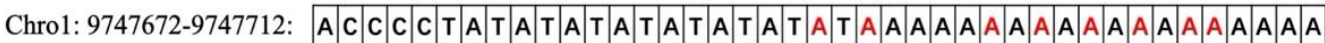

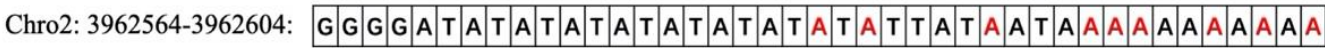

Figure 6. The ROC curves (A) and PRC curves (B) of Deep6mA based on 6mA-rice-Lv dataset.
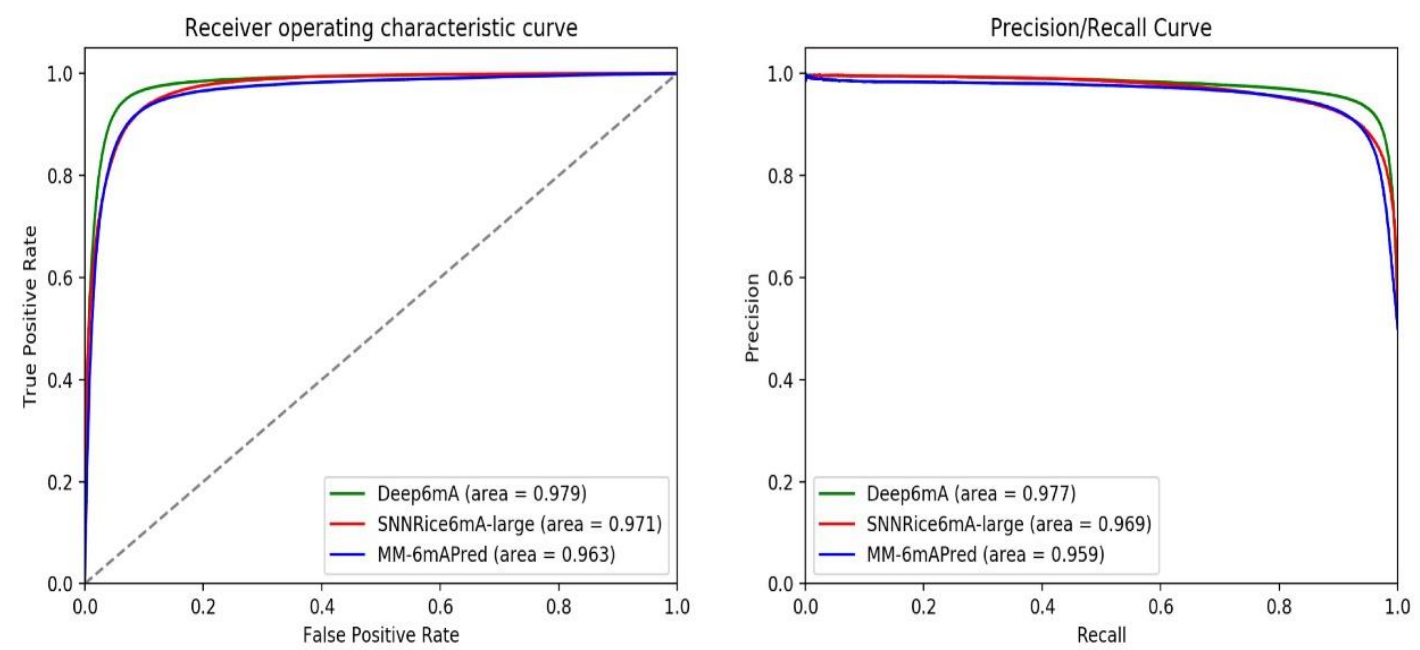

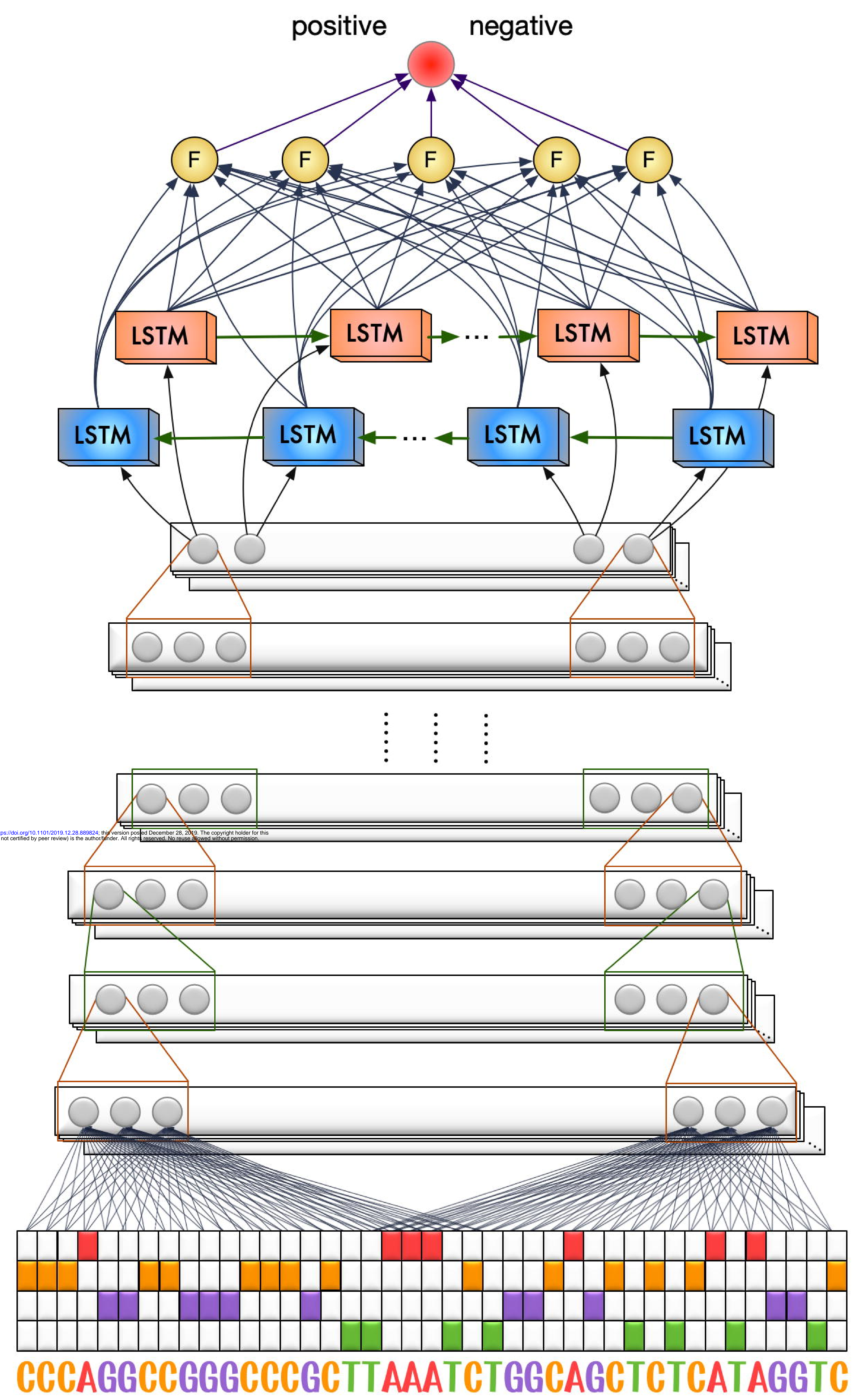

Max pooling<smiles>[C]1CCCC1</smiles>

Convoluton

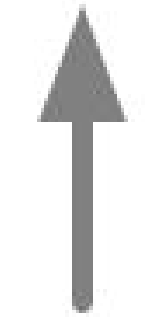

Max pooling

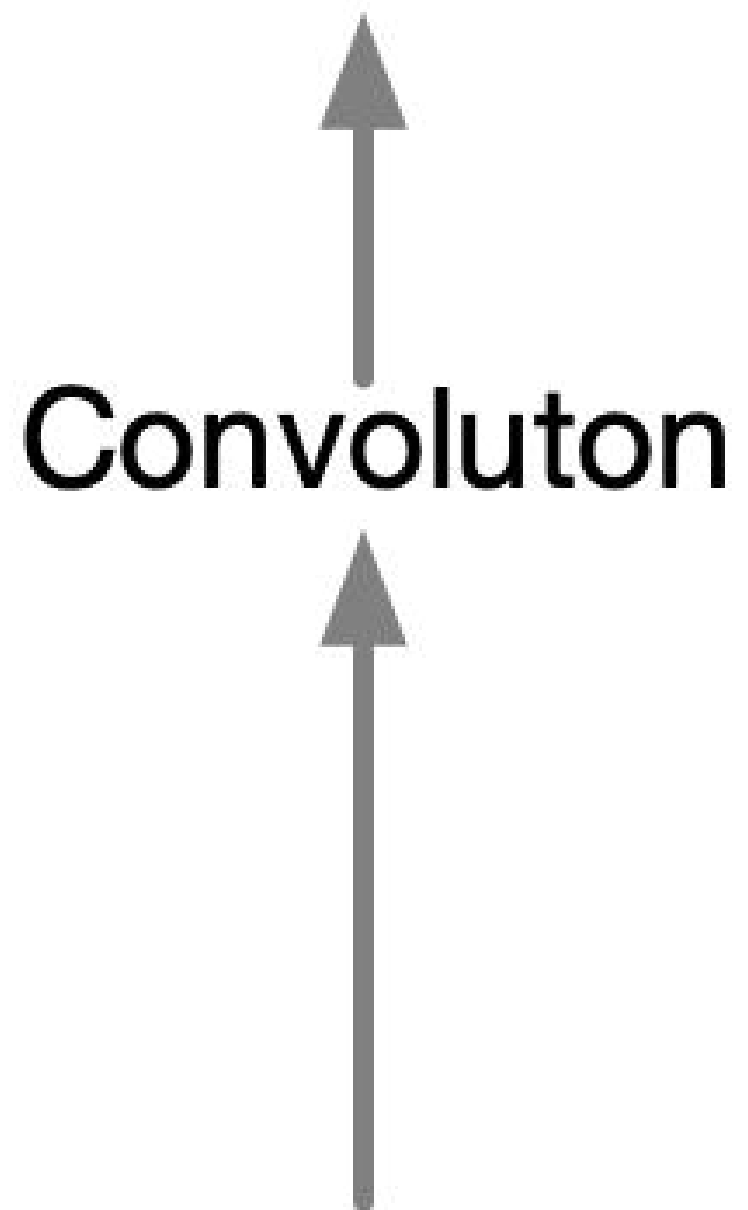

One-hot code 
A

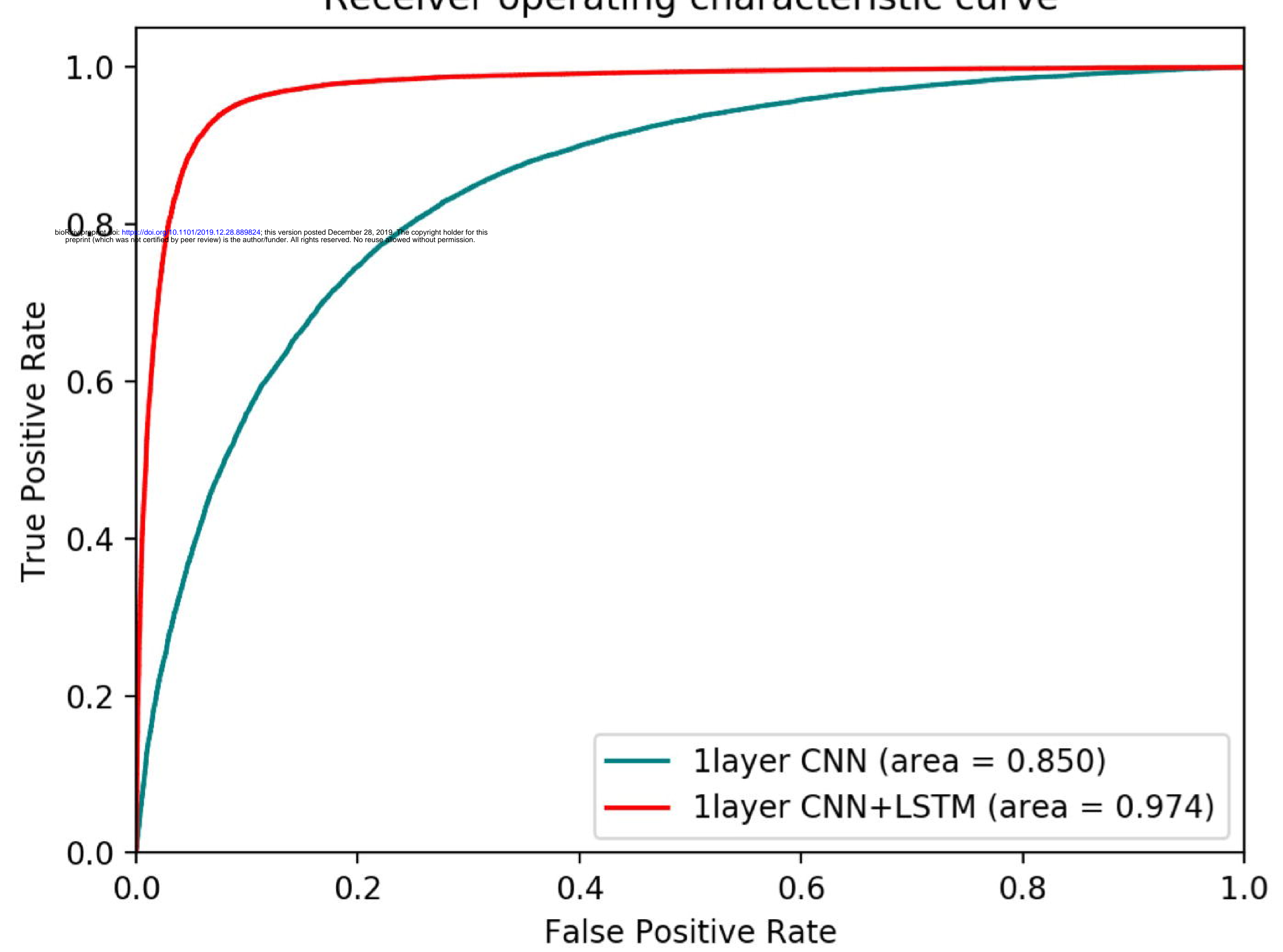

B

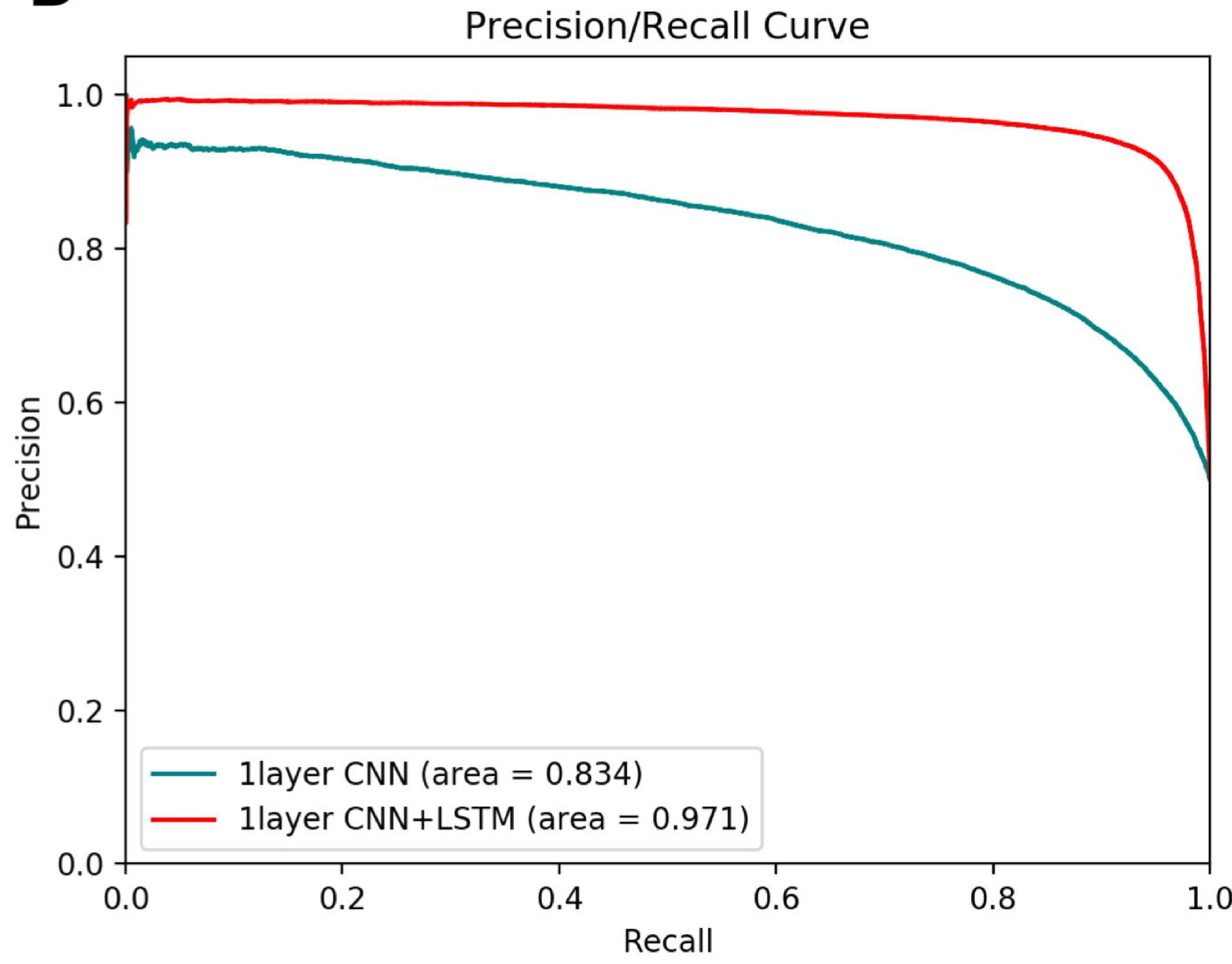


ACC

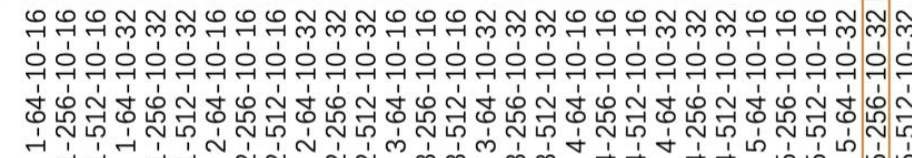

Precision

0.94

0.935

0.93

0.925

0.92

0.915

0.91

0.905

0.9

G GN

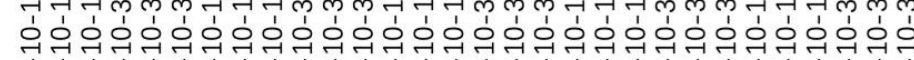
它心
AUC

0.982

0.98

0.978

0.976

0.974

0.972

0.97

0.968

0.966

0.964

0.962

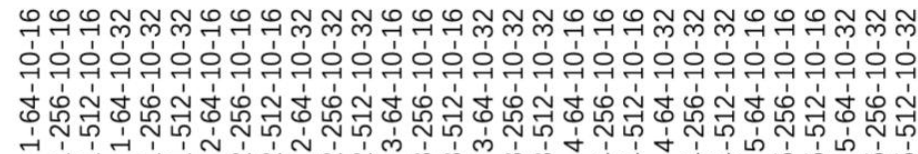

Fscore

0.945

0.94

0.935

0.93

0.925

0.92

0.915

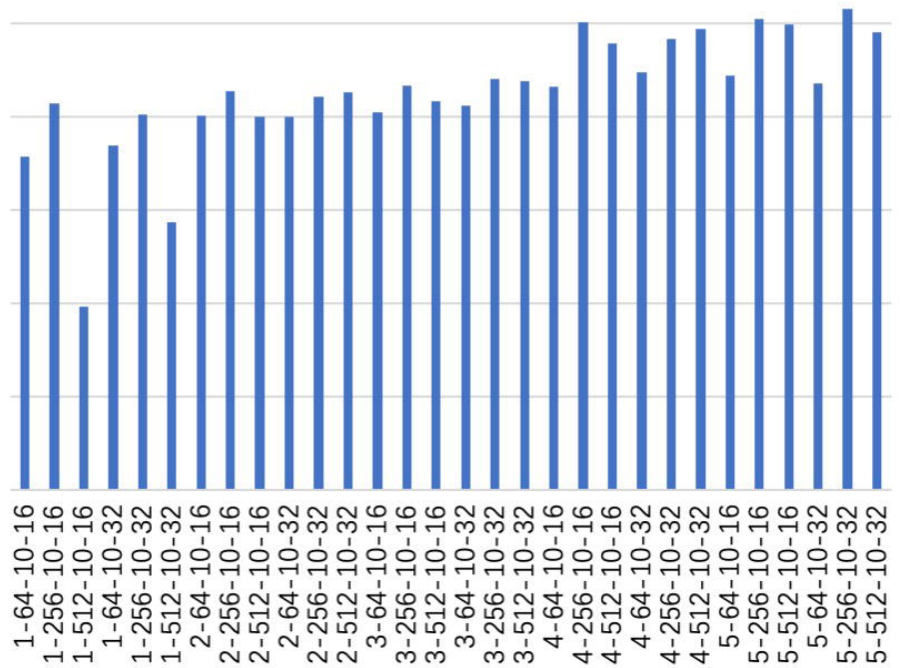

MCC

0.89

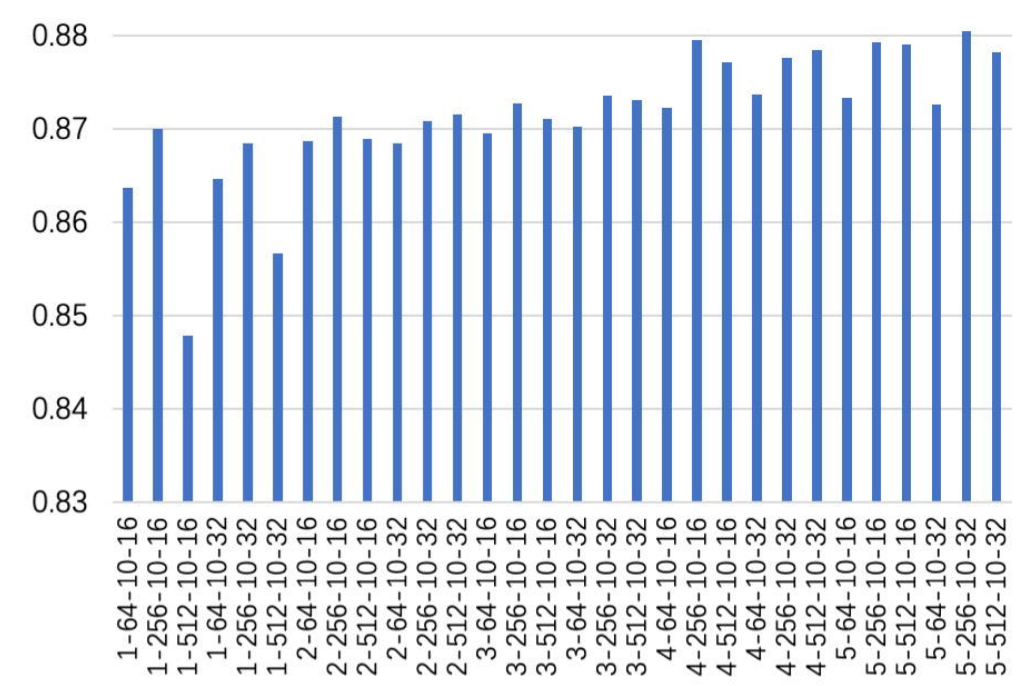

Recall

0.96

0.955

0.95

0.945

0.94

0.935

0.93

0.925

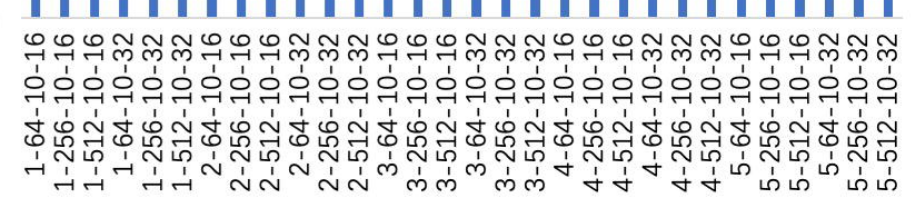


Arabidopsis thaliana
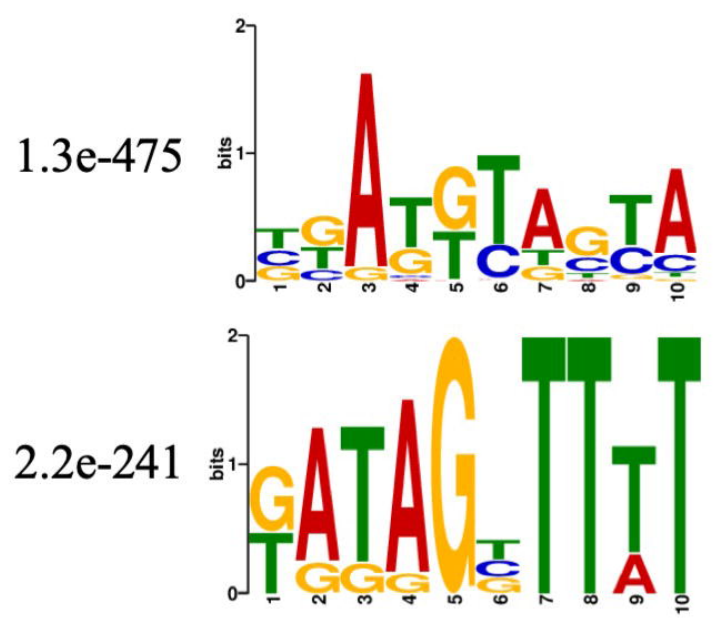

Rice

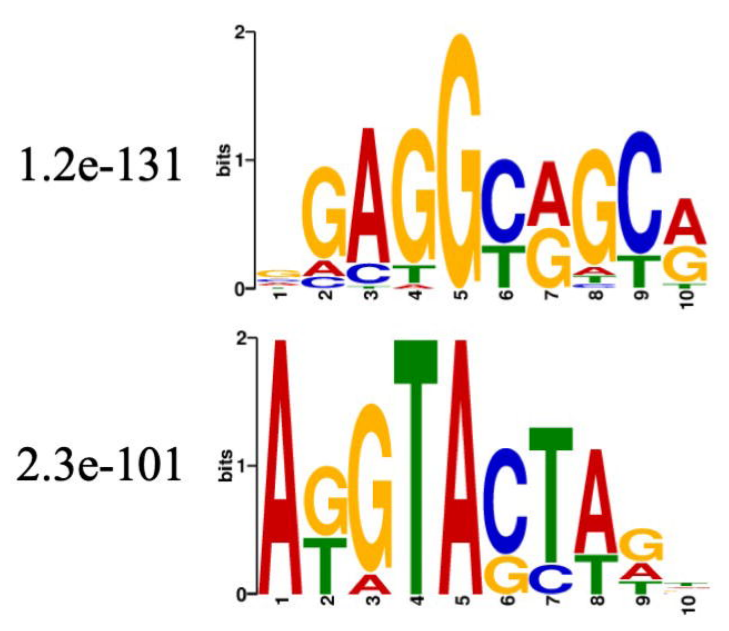

Rosa chinensis

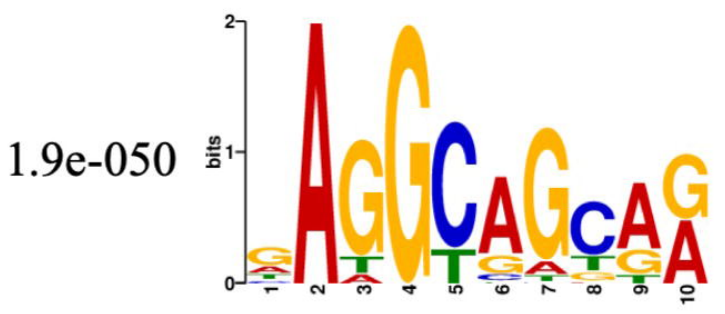

8.1e-005

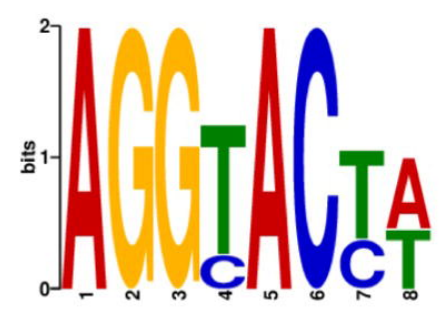

FraVesHawaii

2.3e-039

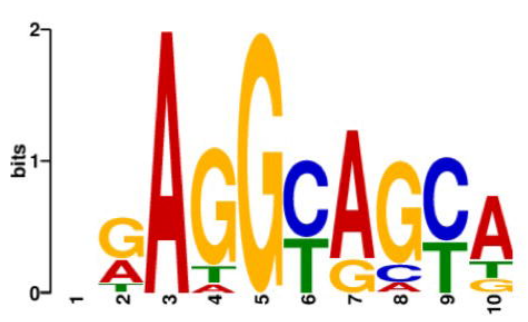

1.1e-003

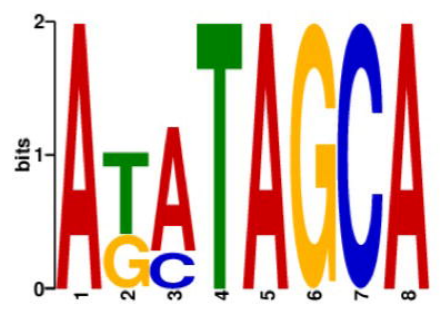




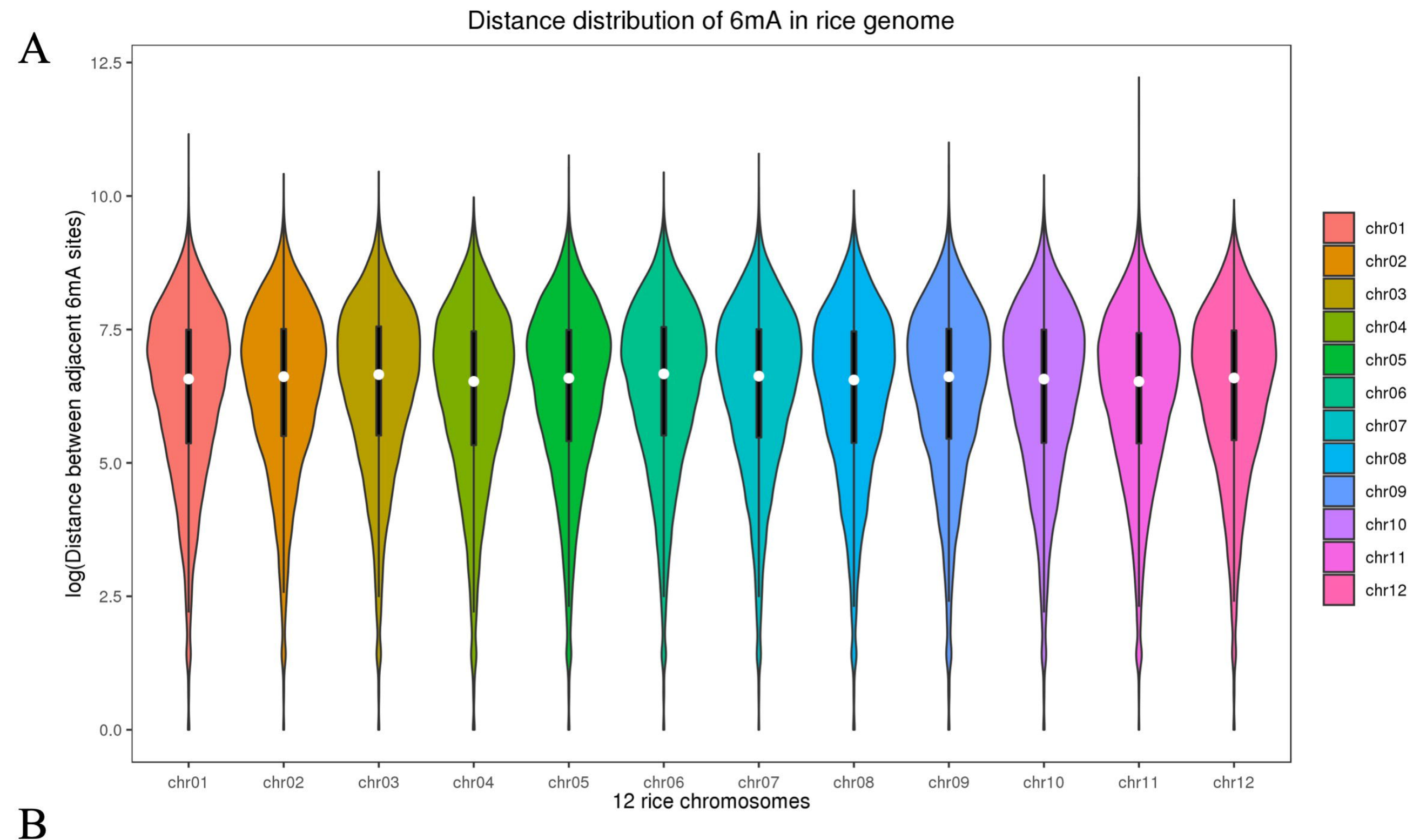

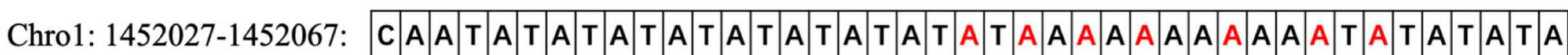

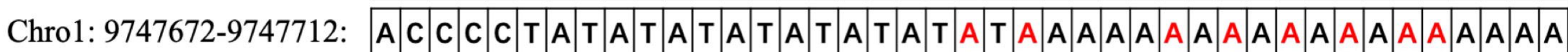

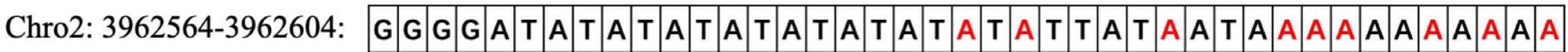



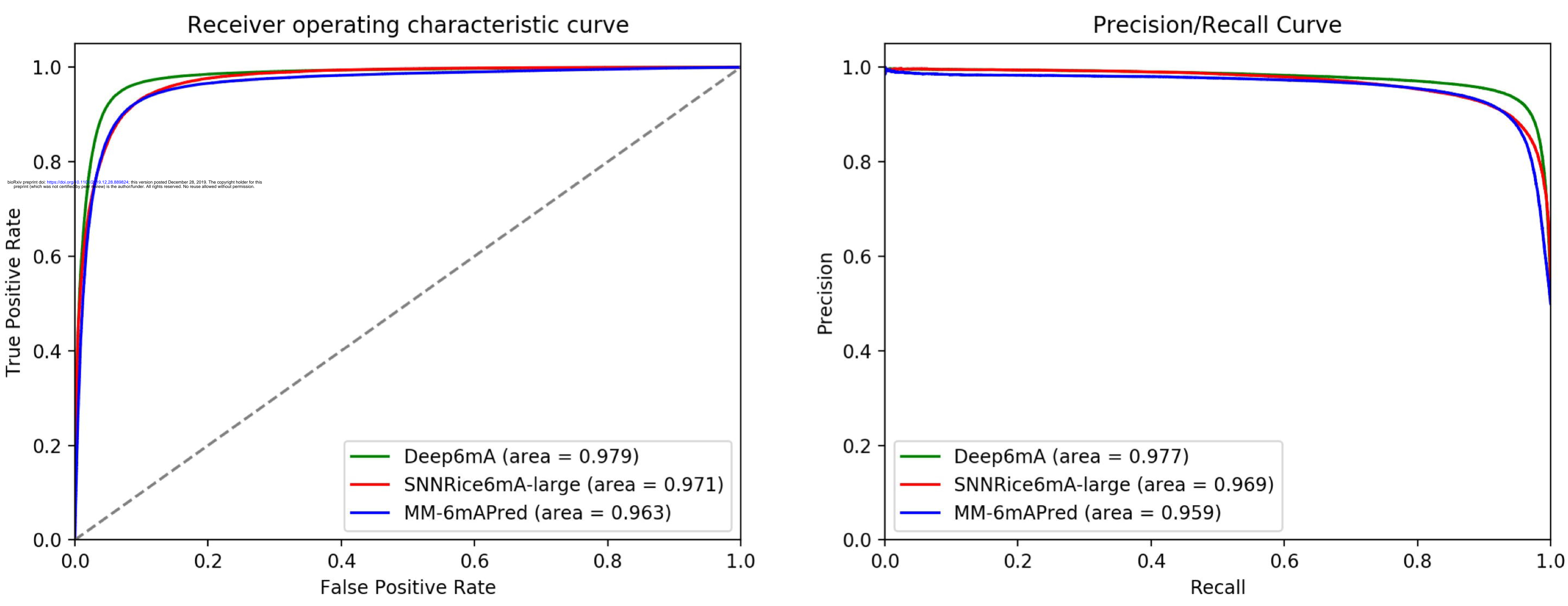